\title{
A Study of Speech Error Phenomena T.O.T Experienced by Advanced EFL Speakers
}

\section{Riyadh Sarhan Al Jebouri}

University of Kufa, Najaf, Iraq

riyadhs.jabr@uokufa.edu.iq

\begin{abstract}
This work is inspecting the tip-of-the-tongue phenomenon, as a sample of advanced EFL users would experience it. It is grounded on the hypothesis that since speaking is a universal process for all human beings, then production models and theories should be universal too, for describing and explaining any phenomenon in any language used whether that language is a native or a foreign language and the occurrence T.O.T. (tip-of-the-tongue) phenomenon in specific. For this reason, advanced EFL users could experience such a phenomenon when using the English language for their every day or their academic life. This paper consists of a theoretical part in which it is considered the basis for defining and explaining the T.O.T. phenomenon and understanding the discussion of the study's expected results. However, in the discussion part, the researcher sheds light on the practical side that contains the questionnaire work analysis, the discussion of the results, and the conclusion that would be expected to be achieved. Based on the above hypothesis while, there were a restricted number of the T.O.T.s; conversely, in such cases, nearly most of the production of speech models and theories that explain the T.O.T. phenomenon is applicable. Concerning the identified T.O.T.s cases, they occurred more with deficient neighbourhoods than words that have sufficient neighbourhoods.
\end{abstract}

\section{Keywords}

Occurrence, T.O.T, deficient and sufficient neighbourhood, EFL users.

\section{Introduction}

Linguistic research has proven that speaking is an extraordinary task of cognitive tasks. Thus, the occurrence of such error during the process of speech production is not surprising. In other words, an ordinary speech is not necessarily free from mistakes, but such mistakes might sometimes be considered to be part and parcel of a normal process of production of speech. Most captivating errors that are caused by what is called a T.O.T.s (tip-of-the-tongue state). At times advanced EFL speakers/users of English think that all of the acquired or learned information in English, especially after a hard studying, is saved in their minds for a long time, and they would be able to retrieve it whenever they need to do so. However, when the time comes to recall the information that they previously learned if that information, whether it is insured in their long-term memories or not, are the learners' most concerning issues. Thus, they may either recall that information easily or not, and sometimes they have the feeling of knowing a particular bit of information, and they experience the T.O.T.s. The
T.O.T.s is numbness of enthusiasm on the verge of retrieving a particular word from the memory when unable to do so (Schwartz, 2002; Brown, 2012). It is mostly supposed to result from the justifications that people make, such as retrieving some of the un-retrieved the attributes of the word, based on other available information (Schwartz \& Metcalfe, 2011). For instance, if a person could not retrieve a word, but he could retrieve its first letter, someone would assume from this that the word is on the edge of access.

\section{The Problem}

Since the user of a language is seen as a universal process shared by all humans, then the speaking production theories should be adequate to explain any language-related phenomenon. The tongue state's tip is acknowledged to be a familiar phenomenon for most speakers of all languages, and it has particular interests for investigating the word retrieval and production. 


\section{The Hypothesis}

It is hypothesized that language production is a universal process implemented by human beings, heretofore the related phenomena of production of a language could be universal. According to Schwartz's universality hypothesis, T.O.T.s would be possible to occur in all participants. A critical outcome to this situation is that learning to read leads to meta-memorial developments, in metacognitive attitude or knowledge curiosity, which would lead to the occurrences of the tongue state's tip.

\section{Objectives and Limitation}

This paper is intended to achieve the following objectives: Investigating the T.O.T.s phenomenon psycholinguistically, discovering the governing factors that incidence of the T.O.T.s, also, and trying to find out whether the EFL speakers experience such speaking incidents. Precisely, do they have the feeling of knowing a word in the English language but cannot retrieve it correctly? Moreover, for checking the applicability of the hypothesis and models of the T.O.T.s that EFL speakers undergo. Therefore, the EFL speakers could experience the T.O.T.s state while they are speaking. The processes of speech production and the theories that easily account for the experimental work results limit this work. Moreover, it is limited to those factors that govern the occurrence of the T.O.T.s. Therefore, it has been limited to some linguistics definitions and character devices previously studied by the participants during their past study experiences.

\section{Literature Review}

The T.O.T.s tip-of-the-tongue state was first hypothesized and studies in psychology (basically in Cognitive Psychology) in 1966 by Brown and McNeill. They read definitions of uncommon words to participants, and they prompted naming problems. The T.O.T.s as a phenomenon has been examined in many experiments since then (Schwartz, 2002), Schwartz put forward evidence that the tip-of-the-tongue experience is linguistically universal in (1999). He has requested fluent native speakers of fifty-one different languages the equivalent of the English expression "to have a word on the tip of your tongue". There was a similar expression for all languages, and in forty-five languages, the temporary failure to recall a word is described as being on or around the tongue. Schwartz construed this oddly high cross-linguistic agreement as reflecting the universality of the phenomenology of the tongue's tip (T.O.T.s); it feels as if there is a word on the tip of someone's tongue and that all humans experience the T.O.T.s. Another theoretical model has been adopted more recently. The Node Structure Theory (N.S.T.) and transmission deficit hypothesis further explain the theorized constructs underlying T.O.T.s and cognition more generally (Burke, 2003). The Node structure theory says that our cognition arises from excitatory priming resulting in activation of cognitive processing units called nodes. The nodes can represent complete constructs like sentences or smaller units such as syllables, and range from meaning to phonetics to the muscle movements required to execute an action (Burke, 1991). The activation of these nodes results in our behaviour, including our speech behaviour. It indicates that the entire action of saying a word is the activation of a combination of nodes required to anticipate and select the word based on its meaning mentally, assemble the required phonological sequence, and form and produce it using our vocal cords, lungs, and mouth muscles. Nodes are organized in a network and function hierarchically.

The top of the hierarchy consists of semantic nodes representing the desired concept, and connections from these semantic nodes unite on the lexical node for the word in question (Burke et al., 1991). Below are phonological nodes representing syllables, compound sounds, and individual phonemes that make up the word in question. Further down the hierarchy at the bottom are nodes for the muscle movements required to produce speech (Burke et al., 1991). The T.O.T. state results from one or some of the nodes in the phonological part of the hierarchy not activating due to weak priming (Burke et al., 1991), coupled using the lexical node's successful activation that represents the word. Some studies have pointed out that maintaining the retrieval processes is mandatory but necessitates 
devoutness resources for the performance. In such studies, the participants are not presented with synchronic tasks to stimulate the detached devotion, but the devotion and concentration performance will be tested. There are various forms of fatigue, like the form that is called central fatigue. Central fatigue fundamentally means, as explained by Allman \& Rice, 2002; an inability to provide the mental processes with enough energy. Central fatigue is a comprehensive concept and can have many causes and indicators.

Nonetheless, when mental processes are affected, language processing might be affected as well. For instance, patients that diagnosed with central or chronic fatigue syndrome often showed speaking and word-finding difficulties. However, such similarity in T.O.T.s is a possible expression between two languages and could reflect phenomenology and the limitations on defining difficult word retrievals' expression. Mostly, when people are in a T.O.T.s the production modality is an oral, and it is mostly natural, hence, that missing words would be stated to as if being on the tongue, without such implying that the word mostly feels as if it is on the tongue. From such a perspective, these expressions should be considered as only as a metaphor. Missing words do not only feel as if they are on a someone's tongue, and the T.O.T.s expressions should, then; not be considered as a reflection of an aspect of the phenomena of a T.O.T.s. To the extent that the universality of T.O.T. phenomena is concerned, if T.O.T.s reveal temporary blocking of lexical access, as in the failure of a universal and fundamental cognitive process, it seems likely that T.O.T.s must be universal. There is an excellent reason to consider that the meta-memory processes play a role in the causes of T.O.T.s, which might be caused to vary.

between cultures or between people with different educational levels. It has shown by Schwartz (1999); that it is quite possible to induce obvious T.O.T.s in contributors for word targets that do not exist, by concealing false queries like "What is the name of Mercury's moon?" within a list of genuine questions. Such illusive T.O.T.s have the qualities of a feeling of incomplete recall and emotionality that genuine T.O.T.s have, although less powerfully
(Schwartz, Travis, Castro, \& Smith, 2000). It shows that people based their judgments of the convenience of recalling, at least in part, on a judgment of in what way it is that they know a word from that particular semantic domain: for instance "I used to read much astronomy, so I should know the answer." Hence the tip of the tongue appears not merely to be an objective index of closeness to recall, but also to reflect a meta-memory judgment. A TOT occurs because there are only single connections to phonological nodes.

In other words, there is one priming connection linking the lexical node to a sound instead of uniting connections, making the phonological part of the network vulnerable to deficits in priming transmission (James \& Burke, 2000). That is why someone in a T.O.T. state often can recall some of the word's sounds, such as syllables or the first letter, but cannot wholly produce it. That is often accompanied by frustration (Burke et al., 1991; MacKay \& Burke, 1996) because the speaker feels so close to producing the desired word. The more often a word is using, the stronger the connections to and from the node become more significant priming (Burke et al., 1991). In addition to novelty and frequency of use, T.O.T.s are sensitive to ageing effects such that as people age, T.O.T.s increase (Burke et al., 1991). That is due to links between all of the nodes in the network become weaker as people age and the phonological system's susceptibility to weakening due to having one-toone connections between lexical and phonological nodes (Burke et al., 1991).

\section{Influencing Factors of T.O.T.s}

The occurrence of the T.O.T.s is varied according to some aspects. These aspects are the word-related factor, the speaker-related factor, and memory.

Word-Related Factor: Certain words are more subjects to the T.O.T.s than others; the variables that could highlight the most essential linguistic and psycholinguistic variables are: 1. Word frequency is an essential variable in all language tasks, and such a frequency always has a "facilitatory effect" 
in speech production. 2. Lexical neighbourhood size refers to some phonologically different words. Some words have sparse neighbourhoods, i.e., words with few or no similar sounding words (e.g., corpse), and others having dense neighbourhoods, for instance, the words with many similarsounding words. 3 . The novelty of a word means that those later ones can quickly and accurately recall a first item or event. 4. Word length, which, straightforward, explains itself (Harley, 1998).

Speaker-Related Factor: The other factor that could influence the occurrence of the T.O.T.s is related to the speaker himself. This factor has two variables, as follow: 1. Brain damage (also called aphasia). 2. Aging attributes to storing and recalling the information weaken because we are getting older.

Memory: The Memory is a processing system, in which the complements of the information-processing approach can be made. The primary stages of processing are 1. Encoding is how information is processed for storage (Phye and Andre, 1986). 2. Storage: is retaining information that is acquired or learned intentionally into memory. All researchers have agreed on the four different memory storage systems, which are: sensory, short-term (primary), working, and long-term memories (secondary). 3. Retrieval: taking information back out when needed. The Retrieval depends on both the quality of the coding and the organization of information transferred to memory (Kagan and Havemann, 1975).

\section{Methods}

The Data collection will be from 20 participants (from Arabic language background who are students at Xiamen University, China. English language is the means of instruction and communication). The age is between 26-37 years old, all males, and postgraduate students. They are going to participate as representatives of advanced EFL users. They have common background knowledge of English had collected from their previous academic studying to a certain level. Such knowledge qualifies them to respond accurately to the questionnaire. The two variables' importance of frequency and length and other variables such as novelty comes from their value in analyzing the questionnaire results.

\section{The Questionnaire and Procedures}

The theory of blocking is more focused on T.O.T.s occurrence and the blocking words nature. It could also accept that weak T.O.T.s might be initiated by a high number or more substantial influence of blocking words and strong T.O.T.s by a weaker influence of blocking words. Consequently, a strong T.O.T. might seem "closer" to the target word because the number or strength of words blocking the path to the target word is low. The partial activation theory is more precise about the relation between T.O.T.s strength and remembrance. This theory proposes that the level of activation is an indication of the T.O.T.s strength. So, if the level of activation is high, the T.O.T.s strength is high as well. So, this study's questionnaire would be modified from (Brennen, Vikan, \& Dybdal, 2007).

The procedure used in this questionnaire is introducing the participants to a questionnaire's consists of two parts about their general and previous knowledge of the English language. They were provided with five linguistics definitions, with each definition, there are the three decisions, "I know," "I do not know" and the "T.O.T.s." These linguistics definitions are selected from the taught/learned sources during their last academic English language study. The definitions are independently listed as from 1-5: Affricative consonant, Labeling, Morphology, Cohesion, and Paraphrasing. The participants who identify the linguistic terms asked to answer it in the blank under and tick "I know." Those who did not know the term asked to make the tick off "I do not know." The participants who are in T.O.T.s, they have been asked to tick with T.O.T.s. For the second part, the participants have to answer as directed in the first part. The correct answers of the second part are: gaze, absurd, dismiss, ignorant and imitate. The purpose of the questionnaire is to check whether the participants are prospective to bring T.O.T.s and the participants who are in the middle to low range of familiarity of definitions that are highly familiar be likely to be recalled rather than to bring a 
T.O.T.s. With participants who have such academic and everyday access to the English language, is it hard to measure what would be established useful items for T.O.T.s bringing?

\section{Data Results}

\begin{tabular}{|l|l|l|l|l|}
\hline No. & $\begin{array}{l}\text { Do not } \\
\text { know }\end{array}$ & Know & TOTS & Total \\
\hline 1. & 6 & 6 & 8 & 20 \\
\hline 2. & 4 & 8 & 8 & 20 \\
\hline 3. & 2 & 14 & 4 & 20 \\
\hline 4. & 4 & 2 & 14 & 20 \\
\hline 5. & 2 & 10 & 8 & 20 \\
\hline Total & 18 & 40 & 42 & 100 \\
\hline
\end{tabular}

Table 1: Results, part 1 of the questionnaire.

\begin{tabular}{|l|l|l|l|l|}
\hline No. & $\begin{array}{l}\text { Do not } \\
\text { know }\end{array}$ & Know & TOTS & Total \\
\hline 1. & - & 14 & 6 & 20 \\
\hline 2. & 4 & 10 & 6 & 20 \\
\hline 3. & 4 & 12 & 4 & 20 \\
\hline 4. & 2 & 10 & 8 & 20 \\
\hline 5. & - & 14 & 6 & 20 \\
\hline Total & 10 & 60 & 30 & 100 \\
\hline
\end{tabular}

Table 2: Results, Part 2 of the questionnaire

\section{Discussion}

For discussing the questionnaire items, the following steps will be considered: The participants to be told that the items were part of a study plan and that it is not required to write the participant's name. The personal details they were requested for were: age, mother tongue, and other languages they could speak and at what age they learned them, how many years they had of academic experiences, and their present situation was?. Then they would be given the questionnaire paper. The participants were then learned that they would now be answered the items about linguistics definitions, some of them were a bit difficult to remember, and some were easy. For any item that they cannot answer, they would be asked whether they knew the answer and whether they were very close to retrieve it. When a participant answered in the affirmative to both of these, it was noted as a T.O.T., the same operationalization of T.O.T.s as discussed in Brown and McNeill (1966). The ten items were read out and in cases of T.O.T.s. That includes the description of the target definition and adjective described in the second part of the questionnaire, and the overall answers of "I know" responses include examining the total number of the "I know" answers in each point. Examined the number of the correct " I know " answers and examined the incorrect answers because of these responses would be considered "T.O.T.s" answers because they are related to the target word either semantically or phonologically. For such answers participant that answered such items did not comprehend that he was in the T.O.T.s. The answer's analysis of "I do not know" such answers analyzed by testing the total answer of the "I do not know" for each point. The "T.O.T. state" answers would have been examining by analyzing the number of the "T.O.T. state" answers which participants ticked for each point.

\section{Analysis and Conclusion}

In analyzing the collected data statistically, it is being listed in order. The first options indicate the "do not know" state, which comes from adding the incorrect responses of the "do know" responses to the "do not know" responses. Number (2) indicates the "T.O.T. state." Moreover, number (3) indicates the correct responses of the "I know" responses.

"Do not know" state "know" state "T.O.T." state

According to this distribution, the mean is "T.O.T. "state. Moreover, the null hypothesis "H0" has been formulated, and the results have been analyzed statistically.

Null hypothesis Ho=TOTs

To verify the study's objectives, the following statistical test was chosen to examine the collected data. 


\section{The Processing Summary- part 1}

\begin{tabular}{|c|c|c|c|c|c|c|}
\hline & \multicolumn{6}{|c|}{ Case } \\
\hline & \multicolumn{2}{|c|}{ Involved } & \multicolumn{2}{|c|}{ Exempted } & \multicolumn{2}{|c|}{ Aggregate } \\
\hline & No. & $\begin{array}{l}\text { Percenta } \\
\text { ge }\end{array}$ & No. & $\begin{array}{l}\text { Percent } \\
\text { age }\end{array}$ & No. & $\begin{array}{l}\text { Percent } \\
\text { age }\end{array}$ \\
\hline TOT & 5 & $100.0 \%$ & 0 & $0.0 \%$ & 5 & $100.0 \%$ \\
\hline $\begin{array}{l}\text { Not- } \\
\text { TOT }\end{array}$ & 5 & $100.0 \%$ & 0 & $0.0 \%$ & 5 & $100.0 \%$ \\
\hline
\end{tabular}

\section{Report}

\begin{tabular}{|l|l|l|}
\hline & T.O.T. & Not T.O.T. \\
\hline Mean & 4.20 & 5.80 \\
N & 5 & 5 \\
\cline { 1 - 2 } Std. Deviation & 1.789 & 1.789 \\
\hline
\end{tabular}

\section{Result}

\begin{tabular}{|c|c|c|c|c|c|c|}
\hline & \multirow[t]{2}{*}{$T$} & \multirow[t]{2}{*}{ Df } & \multirow{2}{*}{$\begin{array}{l}\text { p- } \\
\text { val } \\
\text { ue }\end{array}$} & \multirow{2}{*}{$\begin{array}{l}\text { The } \\
\text { mean } \\
\text { differ } \\
\text { ence }\end{array}$} & \multicolumn{2}{|c|}{$\begin{array}{l}95 \% \text { assertion interval of the } \\
\text { difference }\end{array}$} \\
\hline & & & & & Lower & Upper \\
\hline O & $\begin{array}{l}- \\
2.0 \\
00\end{array}$ & 4 & $\begin{array}{l}0.05 \\
53\end{array}$ & -1.600 & -3.82 & .62 \\
\hline
\end{tabular}

The Participants in part O.N.E. were unable to retrieve more of the definitions they have given, the part O.N.E. answered a mean of 4.2 (SDd 1.789) words, and the second part means of 3.00 (SDd 0.707) words. T.O.T.s were indeed made in the two parts. Data for some items correctly answered Do Know, T.O.T.s, and Do not Know reactions appeared in Table 1. By each PART, the participants have generated T.O.T.s: 4 of the 10. The mean T.O.T. rates for the SECOND part were 3.00, but for part O.N.E. the rates were 4.2. Using the one-tailed test discretely for each PART has shown that the PART ONE answers have significantly increased T.O.T.s. (BOTH PARTS) Have the significance level of Value 0.05 . For the critical finding here that T.O.T.s were for Part One participant that would fail to reject the hypothesis of T.O.T.s is happened for EFL frequently depending on the matters of MEMORIZATION and blocking and sometimes the interference of other linguistics and ecological circumstances such as the culture and environment that the participants are work in/with. It is not meaningful to compare the two parts' T.O.T.s in every one of the groups that had experienced at least one T.O.T. because these events are apt to merely echoing different levels of exposure to the intentioned items, rather than the essential tendency to experiencing T.O.T.s. Also, participants have reported very little partial knowledge of answers targets. A sign of the ITEM length was set for 15 T.O.T.s. Usually, the short items (Adjectives) or long Items were differing in their possibility to remember (DEFINITION). Though, while these were mostly accepted, it is also possible that these reports of word's length are not in fact due to partial lexical access, but rather to reliance on the fact that the adjective tends to be even usually used in a different situation and without thinking ok blocking or any other reason for not retrieve them. Moreover, the definitions might be affected by many causes like the mother tongue, the frequency of using such technical words, and the misunderstanding or short memory situation.

\section{The Processing Summary- PART 2}

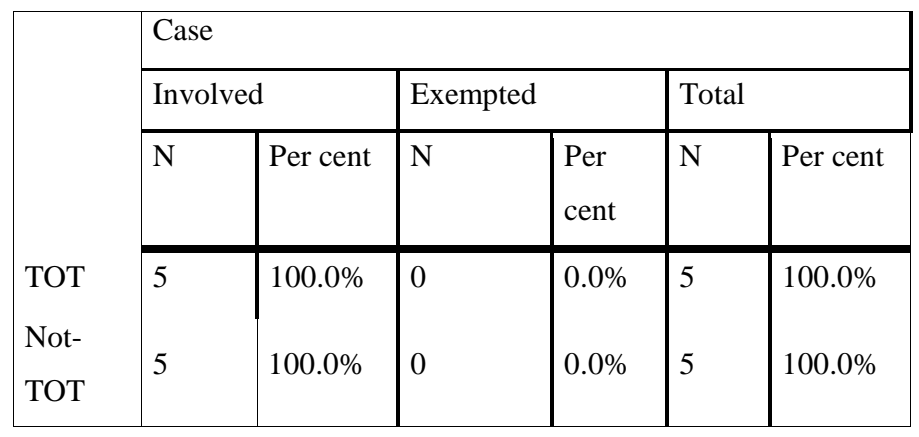

\section{Result}

\begin{tabular}{|c|c|c|c|c|c|c|}
\hline & \multicolumn{6}{|c|}{ The value of test $=0.707$} \\
\hline & \multirow[t]{2}{*}{$\mathrm{T}$} & \multirow[t]{2}{*}{ DF } & \multirow[t]{2}{*}{$\begin{array}{l}\mathrm{P} \text { - } \\
\text { value }\end{array}$} & \multirow[t]{2}{*}{$\begin{array}{l}\text { Mean } \\
\text { difference }\end{array}$} & $\begin{array}{l}95 \% \\
\text { Interval } \\
\text { differen }\end{array}$ & $\begin{array}{l}\text { Confidence } \\
\text { of the }\end{array}$ \\
\hline & & & & & Lower & Upper \\
\hline TOT & 7.251 & 4 & 001 & 2.293 & 1.42 & 3.17 \\
\hline
\end{tabular}


Data in this paper was set to be limited to the idea of the universality of T.O.T.s. It has been thought that the data would make it clear and best to distinguish between the universality of an expression for T.O.T.s and the universality of the phenomenology of a T.O.T.s because the EFL users of language appeared not to have problems with an expression for T.O.T.s. However, sometimes the users report experiencing problems with the phenomenology. T.O.T.s expression refers to temporarily un-retrievable words which would not be seeming to be on the edge of the tongue, but generally, it is in the brain. That would be the result that would suggest if speakers from a specific language background feel like they have a word on their tongue, then this is probably caused by the expression, instead of the other way around. We would argue that the total numbers of T.O.T.s produced in the questionnaire are not a vital issue. Instead, the critical finding is that T.O.T.s could be produced at all in speakers of a language mostly used in spoken or written, including some who are thinking they have mastered the language.

Similarly, the group comparison of how many participants experienced T.O.T.s is not informative because of the item difficulty's misperceiving. If an item is too easy or too complicated for a particular person, it will not generate a T.O.T., so that any differences between participants regarding the T.O.T. state may be due to the level of items difficulty rather than the inherent tendency for experiencing T.O.T.s. The same argument applies to a comparison with the proportion of participants who experience T.O.T.s in Western studies: the issue of item difficulty makes a comparison U.N. interpretable. Nonetheless, we report the number of participants in each group who failed to experience a T.O.T. for the sake of completeness.

The questionnaire has reported that people assume from the presence of a T.O.T. state of the un-retrieved information that they are stable with higher fluency or accessibility information about the target language. When a person in a T.O.T. state, the people judged an un-retrieved word and definitions as more likely to have previously appeared in blocked memory, they also judged these un- retrieved items as more likely be of the word's higher frequency. The analysis of results proposes that the association between stated T.O.T. states, and this analysis not be the result of more fluent or accessible memory representations underlying T.O.T. states than non-TOT states. Therefore; the participants appear to associate the T.O.T. state with an increased possibility that the unretrieved word had more easily accessible qualities. In summary, T.O.T. states themselves seem to discuss the sense of accuracy or fluency on difficult words. During retrieval failure, why should a T.O.T. state lead to the sense of a more fluently accessible word in memory related to a non-TOT state? It is essential to consider that every definition of a T.O.T. state is that the person feels on edge to retrieving a formerly un-retrievable word (e.g., Schwartz, 2001); this implies a sense of better convenience of unretrieved words that produce T.O.T.s were related to unretrieved words that do not have such access. From this viewpoint, it makes sense that people might assume from the presence of a T.O.T. state that the un-retrieved word has qualities that are stable with more critical accessibility. The questionnaire result provides essential perception into recalling the previous knowledge and how it may be affected over time. Especially for those who have started studying science? ( subjects that utterly different from what they used to have as an English language students), However, those in a kind of in touch with the English language literature as people in international relations and administration indicated a significant influence of the occurrence on the number of T.O.T. states produced. The impacts of a single source of instructing interacting led to the results that EFL speakers' prediction may adopt a form of reimbursement to retain proper processing of retrieving the linguistics information.

Schwartz (2002) has proposed that; universality in the context of cultures and languages. It is also interesting to examine it for age. Presently, it is not identified at what age among children the tip-of-the-tongue state expression becomes widely known, but it was evident that preschool children do not do it. However, what about T.O.T. phenomenology: Do younger children experience T.O.T.s? 
Elber (1985) has recorded a T.O.T. in her 2-year-old son. In a conversation of the word "dolphins" in the Dutch language, he frequently retrieves the quite different word for "soldiers". The fun nature of the interaction between mother and son is evident, and it was evident that the child is in an environment that fosters metalinguistic awareness. This study indicates that T.O.T.s might arise relatively soon after the lexicon gets established and that the phenomenology can be experienced many years before the T.O.T. expression is learned.

\section{Conclusion}

To conclude from the above that T.O.T. states result from insufficient activation at the interface between word-forms and sub-lexical representations. Although the present experiments' data cannot directly distinguish between these two accounts, we propose that T.O.T.s are insufficient feedback between word-forms and sub-lexical representations. Identifying the locus of T.O.T.s at the interface between word forms and sub-lexical representations allows us to easily account for our results in an existing model of cognitive processing, namely N.S.T. (Burke et al. 1991, MacKay, 1987), without having to hypothesize modifications or additional assumptions to a model of speech production. In contrast, Harley and Brown (1998). Had proposed that the insufficient feedback between the lemma level (which represents semantic and/or syntactic information) and the lexeme level (which represents the phonological word-form) is responsible for a T.O.T. state.

\section{References:}

[1] Abrams, L. (2008). Tip-of-the-tongue states yield language insights. American Scientist, 96, 234-239.

[2] Biedermann, B., Ruh, N., Nickels, L., \& Coltheart, M. (2008). Information retrieval in the tip of the tongue states New data and methodological advances. Journal of Psycholinguistic Research, 37(3), 171-198.

[3] Brown, A. S. (1991). A review of the tip-of-the-tongue experience. Psychological Bulletin, 109, 204-223.
[4] Brown, R., \& McNeill, D. (1966). The "tip-of-the-tongue" phenomenon. Journal of Verbal Learning and Verbal Behavior, 5, 325-337.

[5] Burke, D. M., Mackay, D. G., Worthley, D. S., \& Wade, E. (1991). On the tip of the tongue: what causes word finding failure in younger and older adults? Journal of Memory and Language, 30, 542-579.

[6] Carroll, D. W. (2008). Psychology of Language. Belmont, CA: Thompson Higher Education.

[7] Choi, H., \& Smith, S. M. (2005). Incubation and the resolution of tip-of-the-tongue states. The Journal of General Psychology, 132, 365-376.

[8] Dell, G. S., Chang, F. and Griffin, Z. M. (1999). Connectionist Models of Language Production: Lexical Access and Grammatical Encoding. Cognitive Science, Vol 23 (4), 517-542.

[9] Elbers, L. (1985). A tip-of-the-tongue state at age two? Journal of Child Language, 12, 353365.

[10] Gollan, T. \& Acenas, L. (2004). What is T.O.T.? Cognate and translation effects on tip-of-the-tongue states in SpanishEnglish and Tagalog-English bilinguals. Journal of Experimental Psychology: Learning, Memory, and Cognition, 30, 246-269.

[12] Harley, T.A. \& Bown, H.E. (1998). What causes a tip-of-thetongue state? Evidence for lexical neighborhood effects in speech production. British Journal of Psychology, 89, 151-174.

[13] James, L. E., \& Burke, D. M. (2000). Phonological priming effects on word retrieval and tip-of-the-tongue experiences in young and older adults. Journal of Experimental Psychology: Learning, Memory and Cognition, 26, 1378-1391.

[14] Janet Metcalfe, B. L. (2017). The tip- of -the- tongue state and curiosity. Springer Open: Cognitive Research: Principles and Implications, 2-31.

[15] Meyer, A. S., \& Bock, K. (1992). The tip-of-the-tongue phenomenon: Blocking or partial activation? Memory \& Cognition, 715-726.

[16] Nussionson, A. K. (2014). What Do We Know When We Forget? In A. S. Brown, the Tip-of-the-Tongue States and Related Phenomena (pp. 327-339). Cambridge: Cambridge University Press.

[17] Roelofs, A. (1999). Phonological Segments and Features as Planning Units in Speech Production. Language and Cognitive Process, pp. 173-200. 
[18] Roelofs, A., Meyer, A.S., \& Levelt, W.J.M. (1998). A case for the lemma/lexeme distinction in models of speaking: comment on Caramazza and Miozzo (1997). Cognition, 69, 219-230

[19] Schwartz, B. L. (2002). The phenomenology of naturallyoccurring tip-of-the-tongue states: A diary study. In S. Shohov (Ed.) Advances in Psychology Research (pp. 7184). Nova Science Publishers: New York

[19] Schwartz, B. L. (1999). Sparkling at the end of the tongue: The aetiology of tip-of-the-tongue phenomenology. Psychonomic Bulletin \& Review, 6, 379-393

[20] Schwartz, B. L., \& Metcalfe, J. (2011). Tip-of-the-tongue (T.O.T.) states Retrieval, behavior, and experience. Memory \& Cognition, 39, 737-749.

[21] Brennen, T. Vikan, A. Dybdal, R. (2007). Are tip-of-thetongue states universal? Evidence from the speakers of an unwritten language. Psychology Press, 15 (2), 167176.

[22] Vitevitch, M. (2002). The influence of phonological similarity neighborhoods on speech production. Journal of Experimental Psychology: Learning, Memory, and Cognition, 28(4), 735-747.

\section{Appendixes}

\section{Questionnaire}

\section{Part One:}

Skim the following explanation and try to answer the following. If you cannot please tick ( ) the letter you are going you use for your answer, and if you are not sure tick ( ) the T.O.T.

Note: T.O.T. means that you cannot remember an entirely correct answer, use this if you feel that you know the answer somehow but cannot remember.

1. A term refers to a sound made when the airpressure behind a complete closure in the vocal tract is gradually released. It is called

a., I know ( ). b. I do not know ( ). c. TOT ( ).

2. A term in a grammatical analysis for the explicit marking of the parts or stages in a structural analysis of a sentence that is referred to...

a., I know ( ). b. I do not know ( ). c. TOT ( ).
3. The branch of grammar which studies the structure or forms of words, primarily through the use of the morpheme construct. It is traditionally distinguished from syntax, which deals with the rules governing the combination of words in sentences, that branch known as......

a., I know ( ). b. I do not know ( ). c. TOT ( ).

4. A term used by the linguists refers to the property of larger units than the morpheme to bind together in constructions, e.g., article + noun. In this use, any group of words which acts as a constituent of a larger unit can be called

a., I know ( ). b. I do not know ( ). c. TOT ( ). 5. A term used in linguistics for the result or process of producing alternative versions of a sentence or text without changing the meaning, this definition refers to.

a., I know ( ). b. I do not know ( ). c. TOT ( ). 6. A term used in some grammatical description models, referring to a class of items whose primary role is to co-occur with nouns to express a wide range of semantic contrasts, such as quantity or number. This is called....

a., I know ( ). b. I do not know ( ). c. T.O.T. ( )

\section{Part Two:}

Do as requested: If you cannot answer, please tick ( ) the letter you are going you use for your answer, and if you are not sure tick ( ) the T.O.T.

Note: T.O.T. means that you cannot remember a fully correct answer, use this if you feel that you know the answer somehow but cannot remember.

1. ........... means are looking steadily, especially for a long time. (Concentrate at, look at, gaze). Choose the correct adjective.

\section{a., I know ( ). b. I do not know ( ). c. TOT ( ).}

2. An adjective that means something unreasonable is (fill the blank) 

a., I know ( ).
b. I do not know ( ).
c. TOT ( ).

3. Write a verb that means setting free and allowing to go. .................... (fill the blank)
a., I know ( ).
b. I do not know ( ).
c. TOT ( ).

4. An adjective that refers to a lack in knowledge, education or consciousness is.

(fill the blank)

a. I know ( ). b. I do not know ( ). c. TOT ( ).

5. .............. is a verb that means copying the behaviour of or appearance or speech of a person. Choose the correct answer from (imitate, copy, parrot)

a., I know ( ). b. I do not know ( ).

c. TOT ( ). 